\title{
Thoughts on breast cancer in Brazil
}

\section{REFLEXõEs SOBRE O CÂNCER DE MAMA No BRASIL}

\author{
Carlos Alberto Ruiz ${ }^{1}$, Ruffo Freitas-Junior ${ }^{2}$
}

1President of the Brazilian Society of Mastology (2011-2013)

2President of the Brazilian Society of Mastology (2014-2016)

In Brazil, a continuous increase in the incidence of breast cancer has been observed, with the projection of over 57,000 new cases in 2014. Unfortunately, the mortality projection due to this type of cancer in the country is also high, and approximately 13,000 Brazilian women will have their lives cut short by the disease.

Approximately $30-40 \%$ of breast cancers diagnosed in this country are detected with sizes above $3 \mathrm{~cm}$, also with axillary node involvement by the tumor. This finding is important because breast cancer takes on average 8 to 10 years to reach $1 \mathrm{~cm}$, which in principle would allow us sufficient time for early diagnosis. From this dimension, if nothing is done, it can lead to death within three years, but the patient rarely dies of local problems, but as a result of metastases, mainly to bones, liver, lungs, and eventually to the central nervous system. The risk of metastasis is known to be directly proportional to tumor size.

This makes early diagnosis critical. Diagnosis at an early stage and proper treatment can provide up to $95 \%$ chance of cure. However, to achieve these numbers, it is important that mammograms are performed annually from the age of 40 years, which may have an impact on mortality reduction of up to $35 \%$, as demonstrated in large controlled studies. Other major advantages of early diagnosis are that in most cases there will be no need to remove the breast and the indication for chemotherapy will be reduced.

The modern treatment of breast cancer is complex and customized, requiring surgery with oncologic and aesthetic refinement, radiotherapy, chemotherapy, endocrine therapy and immunotherapy with monoclonal antibodies. Access to all these treatment modalities ensures the best possible results, while if any of these procedures is not done properly, the others may be compromised, leading to less effective results.

In Brazil, there are approximately 18,000 women over the age of 40 years who in theory would be entitled by law to receive yearly mammograms. Nevertheless, only $5 \%$ to $30 \%$ of them get mammogram screening under the Unified Health System (SUS), which on average in the coun- try does not quite cover $25 \%$ of what it should. There is no qualification, access, information, education, investment, structure or willingness.

When comparing mortality from breast cancer between developed countries and developing countries we observe an increase of $40 \%$ on the latter, indicating that being born in a poor country leads to significant increase in mortality, which is the sad situation seen in Brazil.

While the mortality rate decreases in developed countries, including the US, UK and many others, recent work conducted by the Mastology Research Network of Goiás indicates that mortality from breast cancer in Brazil is stable over the past 15 years, with an incredible inequality between states, which is also reflected in the country's macro-regions. Mortality continues to grow in Brazil's North, Northeast and Midwest, while the reduction in mortality is already perceived in the Southeastern and Southern regions, probably due to greater access to treatment and, especially, early diagnosis. In these two regions, locally advanced tumors are less frequent than in other regions. Investing in structure is fundamental to everything in health, especially in relation to chronic diseases, which includes breast cancer. The problem is not a shortage of doctors, but the lack of infrastructure and adequate diagnostic and treatment centers. There is no plan to secure trained doctors in small towns and not suitable conditions for good service in most cities in the interior of this vast country.

What we see when we look at what happens in our country, comparing the capital cities and the interior in terms of mortality rate, is identical to what we see when comparing developed and developing countries. That is, compared to the country side, the chance of cure is higher in state capitals, except for the state of São Paulo.

Another big difference is in health standards in Brazil; comparing private medicine with public medicine we find even more striking differences. A recent study by the Gbecam group (Amazona Project) pointed out that the diagnosis of breast cancer occurs at a later stage in the public service and, as a result, more women die from breast cancer in public services than in private. The authors 
found that patients treated by the SUS are also subjected more often to mutilating surgery, because they have more advanced tumors. Furthermore, progression-free survival, overall survival and survival after first progression are all lower in SUS patients, which shows the unacceptable disparity in access to early diagnosis.

On the other hand, when there is access to early diagnosis for the poor, the survival rates between the public system and the private system resemble. In other words, being poor in a country like ours is to suffer more, to survive less, and to be more mutilated. This situation shames us all.

What kind of health is this?

The complexity arising from the screening and treatment of breast cancer is higher because of all this. We need to spread the idea and the strategies that benefit early diagnosis, that is, we need to encourage and achieve early detection more and more. The Brazilian Society of Mastology (SBM), the Brazilian College of Radiology (CBR) and the Federation of Brazilian Societies of Gynecology and Obstetrics (Febrasgo), in a joint guideline, recommend annual mammography starting at age 40 . This is the recommendation of experts of three medical-scientific entities, and a legal right of women in this country. Ensuring this right means less mutilation, less aggression and achieving survival rates similar to those in developed countries, offering more dignity to women, especially those in worse financial condition in this fabulous country that we love so dearly. 